\title{
THE USE OF WORKING MEMORY FOR TASK PREDICTION: WHAT BENEFITS ACCRUE FROM DIFFERENT TYPES OF FOREKNOWLEDGE?
}

\author{
J. J. S. BARTON, ${ }^{a, b *}$ A. KUZIN, ${ }^{b}$ F. POLLI $\left.\right|^{b, c}$ \\ AND D. S. MANOACH ${ }^{\mathrm{b}, \mathrm{c}, \mathrm{d}}$ \\ a Departments of Neurology, Ophthalmology and Visual Sciences, \\ Neuro-ophthalmology, Section D, VGH Eye Care Center, University of \\ British Columbia, 2550 Willow Street, Vancouver, BC, Canada V5Z \\ 3N9 \\ ${ }^{b}$ Harvard Medical School, Boston, MA, USA \\ ${ }^{c}$ Department of Psychiatry, Massachusetts General Hospital, Boston, \\ MA 02215, USA
}

${ }^{d}$ Athinoula A. Martinos Center for Biomedical Imaging, Charlestown, MA 02129, USA

\begin{abstract}
The assumption that the deployment of executive processes invariably improves task performance is implicit to cognitive theory. In particular, working memory can be used to retain and update historical information about predictable trial sequences (foreknowledge) so that subjects can anticipate and prepare for the upcoming trial more effectively. We review the effects of different types of foreknowledge on response accuracy and latency, particularly in relation to experiments investigating saccadic eye movements in humans. While it is possible to make all aspects of an impending trial predictable, varying the predictability of different components of the trial independently can reveal which cognitive operations are potentially modifiable by foreknowledge. These operations include stimulus processing, retrieval of task-set rules, and response preparation, among others. The available data suggest that, while response preparation can be completed and the response even executed before the stimulus appears (i.e. anticipation) when the subject possesses complete taskforeknowledge (knowing both the stimulus to appear and the response required), foreknowledge of the task-set alone does not permit advance configuration of the task-set rules. A taxonomy for foreknowledge is proposed, including foreknowledge for timing, stimulus, set, response, and task. Work on differentiating these effects in neurophysiology, neuroimaging, and neuropsychology is still in the early stages. () 2005 Published by Elsevier Ltd on behalf of IBRO.
\end{abstract}

Key words: working memory, foreknowledge, saccade, prediction, task-switch.

Working memory is the process of actively holding information 'on-line' in the mind's eye and using that information to guide behavior (Baddeley, 1992). It is a temporary store whose contents are continually updated, scanned and manipulated in response to immediate information processing demands. It is a building block of normal cognition that is critical for higher cognitive functions and

*Corresponding author. Tel: +1-604-875-4339; fax: +1-604-875-4302.

E-mail address: jasonbarton@shaw.ca (J. J. S. Barton).

Abbreviations: fMRI, functional magnetic resonance imaging.

$0306-4522 / 06 \$ 30.00+0.00$ @ 2005 Published by Elsevier Ltd on behalf of IBRO. doi:10.1016/j.neuroscience.2005.10.001 goal-directed behavior. In the present review, we consider the benefits that accrue, or fail to accrue, from using working memory to predict and prepare for upcoming tasks.

While responding to a simple single task may become fairly automatic with practice and may make minimal demands on working memory, having to switch between tasks requires greater working memory to maintain more than one task-set in a state of readiness. In task sequences that involve a non-random sequence of tasks, working memory is necessary to discern the pattern of task occurrence and to use this information to predict and prepare for an upcoming trial. Pattern recognition requires that recent trial history be held in mind and prediction requires that this pattern of trials be used to anticipate what will occur next.

Prediction, or foreknowledge, can enhance behavior. For the purposes of this review, we define foreknowledge as the derivation of completely reliable information about some or all properties of an upcoming trial from the historical context of previous trials. We distinguish this from advance information due to explicit cues in the early portion of the trial (pre-cueing). Although some investigators also label pre-cueing a form of foreknowledge (Schiller et al., 2004), pre-cueing does not require use of information from trial history. Foreknowledge from trial history can be considered a special subset of situations in which the prior probabilities of trial properties are varied to study the effects of expectation (e.g. Carpenter and Williams, 1995; Dreisbach et al., 2002; Schiller et al., 2004). Foreknowledge is the special situation where the prior probability of something occurring in the upcoming trial is 1 . While this review focuses upon foreknowledge with complete certainty about some aspect of the trial, we include data from studies of prior probability effects where they are relevant. Advance knowledge about the probabilities or properties of the upcoming trial is not necessary for task performance in many paradigms (for an exception, see Koch, 2003), but may allow some degree of preparation, which presumably could improve task execution.

If a paradigm involving a sequence of trials is predictable only in that all trials are identical, this places minimal if any demands on working memory. However, to exploit predictability in a paradigm involving a sequence of different trial types requires both maintenance of the sequence in working memory and updating of the current position in the sequence with each trial. Presumably, the longer the sequence is the greater the demand on working memory, up to a point where the capacity of the system is reached. Within its limits, though, the deployment of working mem- 
ory with predictable sequences should result in better performance than when it is not utilized. This type of paradigm may be a particularly useful way of assessing working memory efficacy, since it allows the effects of working memory to be manifest as an improvement over a baseline condition in which it is not operating, such as a block with a random, unpredictable sequence of trials.

In this review we first discuss the various types of foreknowledge that can be examined. These can be divided into foreknowledge for different trial aspects that affect different cognitive stages in the generation of behavior, including stimulus processing, readying task set rules for responses, and response execution and synchronization. We propose a taxonomy based on these distinctions, that may guide investigations of the role of foreknowledge in cognition (Table 1). Next, we examine in more detail the data on set-foreknowledge, which have shown that the process of reconfiguring task sets is surprisingly immune to the effects of foreknowledge. Last, we review the current state of foreknowledge in the fields of neurophysiology, functional imaging, and neuropsychology, which we hope will illuminate the value of a taxonomy and the need for further studies of the role of foreknowledge in guiding behavior. This is a relatively under-developed area and the aim of this review is to provoke further investigations.

\section{Varieties of foreknowledge}

Many different aspects of a trial can be made predictable. At one extreme, one can have complete foreknowledge of both the stimulus and the response required. On tasks requiring saccadic responses to peripheral targets, for example, it may already be known from the trial sequence that the next stimulus will appear on the left at 10 degrees and that the required response is a leftward saccade (e.g. Moschner and Zangemeister, 1993). We call this 'complete task-foreknowledge,' since the effect of such complete predictability is that the subject knows before the trial begins both what stimulus will appear and what specific

Table 1. A taxonomy of foreknowledge

\begin{tabular}{|c|c|}
\hline $\begin{array}{l}\text { Stimulus } \\
\quad \text { foreknowledge }\end{array}$ & $\begin{array}{l}\text { Knowing which stimulus will occur, but } \\
\text { without knowing what rule to use to } \\
\text { generate the response, and therefore not } \\
\text { knowing the specific response needed }\end{array}$ \\
\hline Set foreknowledge & $\begin{array}{l}\text { Knowing what rule to use to generate a } \\
\text { response, but without knowing which } \\
\text { stimulus will occur, and therefore not } \\
\text { knowing the specific response needed }\end{array}$ \\
\hline $\begin{array}{l}\text { Response } \\
\quad \text { foreknowledge }\end{array}$ & $\begin{array}{l}\text { Knowing the specific response needed, but } \\
\text { without knowing which stimulus will } \\
\text { occur, and therefore not knowing the rule } \\
\text { that generated that specific response }\end{array}$ \\
\hline $\begin{array}{l}\text { Complete task } \\
\text { foreknowledge }\end{array}$ & $\begin{array}{l}\text { Knowing which stimulus will occur, knowing } \\
\text { what rule to use to generate the } \\
\text { response, and therefore knowing the } \\
\text { specific response needed }\end{array}$ \\
\hline $\begin{array}{l}\text { Selective task } \\
\text { foreknowledge }\end{array}$ & $\begin{array}{l}\text { Task foreknowledge in which some } \\
\text { stimulus aspects are known but not } \\
\text { others (e.g. amplitude but not direction) }\end{array}$ \\
\hline $\begin{array}{l}\text { Timing } \\
\text { foreknowledge }\end{array}$ & $\begin{array}{l}\text { Knowing exactly when the stimulus will } \\
\text { appear }\end{array}$ \\
\hline
\end{tabular}

response they will have to make. This foreknowledge can allow subjects to prepare the exact response in advance of the stimulus, leading to shortened latencies and increased frequencies of express saccades (Kingstone and Klein, 1993; Paré and Munoz, 1996; Schiller et al., 2004; Wegner and Fahle, 1999). When the timing is also predictable, there are also large numbers of anticipatory responses, which either precede the target or occur too soon after its appearance to have been triggered by the target (Moschner and Zangemeister, 1993). Clearly the use of working memory to store information about the sequence of specific required responses can allow the subject to more efficiently prepare and sometimes prematurely execute the complete saccadic response.

Related to this concept of complete task-foreknowledge are a number of prior probability phenomena. For example, in some tasks response latencies are correlated with the number of potential stimuli (and hence responses) in the array. This would suggest that some processing efficiencies are possible when readiness can be limited to a smaller set of responses, even if this is not limited to a single stimulus and response as in complete task-foreknowledge. This effect may be due to the fact that with fewer targets, each target has a higher prior probability of appearing. Whether this is true of the number of target locations in our saccade example is a matter of debate (Michard et al., 1974; Saslow, 1967). However, a relation between saccadic latency and number of stimulus locations appears to exist when the task includes distractor stimuli (Viviani and Swensson, 1982). In a more direct example of the effects of prior probabilities on saccades, changes in the frequencies at which saccadic targets appear result in shorter latencies and more express saccades to high-probability targets than to low-probability ones (Megaw and Armstrong, 1973; Schiller et al., 2004). Indeed, it has been shown that saccadic latencies vary inversely with the logarithm of the prior probability of the target and its required response, consistent with a model of decision-making and decision-signal rise time based on likelihood theory (Carpenter and Williams, 1995).

It is also possible to vary the predictability of different stimulus and response parameters independently. Paradigms that provide incomplete or 'selective task-foreknowledge' can be informative about the type of response programming that can benefit from advance preparation. In the context of simple saccadic responses, the direction, amplitude and timing of the target and its required saccade can all vary in their predictability. An early study found that the probability of target direction (left versus right) significantly influenced the latency of the response, even if target amplitude was variable (Megaw and Armstrong, 1973). On the other hand, the predictability of the amplitude of the target step did not offer further advantages to latency when the target direction was already known. The authors concluded that response selection was primarily organized by direction within a plane, rather than amplitude. (However, the far greater spatial separation between the directiondiffering stimuli versus the amplitude-differing stimuli in this 
study is a confounding factor that raises doubt about the validity of this conclusion.)

In addition to the spatial metrics of targets, time of onset is another stimulus variable that can be made predictable or unpredictable. Providing a rhythm to trials allows a subject to better synchronize task-related processes to target onset, but the benefits of this synchronization may vary with the preparatory states of these different processes. Hence 'timing-foreknowledge' may be a 'co-factor' that secondarily modulates the effects of other foreknowledge: that is, its effects may stem from interactions with other types of task foreknowledge. For example, one saccade study found very few anticipatory saccades when the task contained only timing-foreknowledge or only task-foreknowledge, but a large number of anticipatory saccades when both timing-foreknowledge and task-foreknowledge were present (Moschner and Zangemeister, 1993). Similarly, another study found no increase in anticipatory saccades with timing-foreknowledge alone, but more with either taskforeknowledge or both task- and timing-foreknowledge (Gagnon et al., 2002). (This is not to say that anticipatory saccades are never found with only timing-foreknowledge. When subjects are instructed to respond as rapidly as possible in a task with predictable timing, they may produce anticipatory saccades even when the desired response is not known (Kalesnykas and Hallett, 1987). These are guessing saccades, with accuracy rates close to chance.) For regular prosaccades, the presence of both timing- and task-foreknowledge also led to a greater reduction in latency than when either alone was present (Gagnon et al., 2002). On the other hand, timing-foreknowledge does not seem to benefit responses in taskswitch paradigms, whether set-foreknowledge (see below) is present or not (Dreher et al., 2002).

In the examples given above, the rules for responding to the target remain constant, so that once certain properties of the stimulus are known, the corresponding properties of the required response are also known. Therefore selective foreknowledge about the stimulus (e.g. it will appear on the left in $3 \mathrm{~s}$, but at a variable distance) translates directly into a similar selective foreknowledge of the response (e.g. in $3 \mathrm{~s}$ I will need to make a leftward saccade of uncertain amplitude). However, other partially predictive paradigms can be constructed such that there is foreknowledge of either the stimulus type or the response type but not both. For example, one might know what type of stimulus will be shown but not the exact response required. Thus, in a saccadic paradigm one might have correctly predicted from the sequence of trials that the next target will be on the left, but still be uncertain until a later cue as to whether the response should be a prosaccade (look at the target) or an antisaccade (look opposite to the target). This then would be merely 'stimulus-foreknowledge.' Another possibility is that one might not know which stimulus will appear, but know the type of response that will be required. In the saccadic paradigm, one may then be uncertain as to which side the target would appear, but know from the trial sequence that an antisaccade was the required response (Barton et al., 2005; Sohn and Anderson, 2001; Tornay and Milan, 2001). This would be a 'set-foreknowledge.' In contrast to complete task-foreknowledge, where the exact saccade required is known ahead of time ('look left'), in set-foreknowledge only the task-set rule, or set of responses, is known ('if target is at X, then look 180 degrees opposite'). A third, more unusual, possibility is that one might know the specific response required ('look left') without knowing which stimulus and task-set rule will be used to generate it (i.e. will it be a left target with the prosaccade rule, or right target with the antisaccade rule?). This 'response foreknowledge' has seldom been examined.

Investigations of these types of partially predictive trial sequences can allow us to determine what aspects of the cognitive processing from stimulus to response can exploit the benefits of foreknowledge as deployed through working memory. Can stimulus-foreknowledge circumvent the need for stimulus processing, other than the mere triggering function of its onset? Does set-foreknowledge allow subjects to prepare the set-rule in advance of the stimulus? Are these advantages reflected in accuracy changes, latency changes or both?

The effects of selective stimulus-foreknowledge have seldom been investigated. One study of prior probabilities has provided evidence that the allocation of attention to a stimulus can be modified by the likelihood of that stimulus occurring (Myles-Worsley et al., 1991). This study presented a series of word pairs, one word above the other, flashed too briefly for both to be read. At random points in the series the subject was asked to name the word just presented at one of the positions chosen at random. When the word at one position was made more predictable, the accuracy of identification of the word at the other position increased, indicating that attention was directed away from the more predictable location even though the subject did not know which position would be interrogated for the response. This result suggests that stimulus processing can be affected by foreknowledge, independent of foreknowledge effects on response preparation.

\section{Experiments in set foreknowledge: does prediction always help?}

Our recent investigations have focused on the benefits of set foreknowledge in a saccadic paradigm (Barton et al., 2005). Set-foreknowledge in particular is revealed during instances where the set changes from one trial to the next. Contrasts between trials preceded by similar sets and trials preceded by different sets can reveal the effects on latency and accuracy that are due to reconfiguration from one set to another. This involves both disabling the previously operating set rule and enabling the new set rule required in the current task.

Studies that vary the interval between an instructional cue and the appearance of a target stimulus have established that there is a significant component of set-reconfiguration that can be executed in advance of stimulus onset (Meiran, 2000). This is the conclusion from findings that the difference in latencies between switched and repeated trial types (the latency switch cost) can be reduced by having the instructional cue precede the target by a 
second or more. The assumption is that this latency reduction reflects the time needed to execute this component of the cognitive operation of set-switching. Because this latency switch effect depends on the temporal relation between the cue and the target stimulus, rather than the temporal relation between the prior trial and the current stimulus, it is considered an active switching process rather than a passive inhibitory or facilitative carry-over from the set in the prior trial (Meiran, 1996; Meiran et al., 2000). This active 'advance reconfiguration' is of particular interest for study of the effects of set-foreknowledge. If advance reconfiguration can be triggered by an instructional cue that precedes the stimulus, can it also be triggered in advance of the stimulus by set-foreknowledge?

To study this, we recently performed an experiment in which subjects performed blocks of trials that contained mixtures of prosaccades and antisaccades (Barton et al., 2005). In one group of blocks the mixture was randomized. In the other the subjects were told that the sequence of task sets would follow a predictable four-trial sequence of two prosaccades followed by two antisaccades. Again, to use this predictive information subjects had to use working memory to maintain the sequence and update their position in the sequence for each trial. The predictive information was redundant to the performance of the task, as each trial contained an explicit instructional cue stating whether a pro- or antisaccade was required in each trial. This was a cue at fixation that was either a blue $X$ or a yellow ring. The critical parameter was the amount of advance preparation the cue provided. In one group of blocks the cue occurred a mere $200 \mathrm{~ms}$ before the target stimulus, affording little preparation: thus the response latencies in these trials would also include most of the time needed to reconfigure the set, if a switch of set was called for. In the other group of blocks the cue occurred $2000 \mathrm{~ms}$ prior to the target, long enough to allow reconfiguration for switched trials in advance of the imperative stimulus (Meiran, 1996; Rogers and Monsell, 1995).

If subjects were able to use set-foreknowledge to reconfigure their set from prosaccades to antisaccades or vice versa, then the amount of advance warning given by the instructional cue should not matter in the blocks with a predictable set sequence. That is, predictable sequences with a $200 \mathrm{~ms}$ cue-target interval should give latency switch costs no different than predictable sequences with a 2000 ms cue-target interval. Also, the amount of advance reconfiguration with predictable sequences should be similar to the advance reconfiguration permitted by a long cue-target interval. Hence predictable sequences with either $200 \mathrm{~ms}$ or $2000 \mathrm{~ms}$ cue-target intervals should yield switch latency costs similar to random sequences with a long 2000 ms cue-target interval.

These were not the results we obtained (Fig. 1). We did find, as expected, that for random sequences the latency switch costs were higher for short cue-target intervals than for long cue-target intervals. This demonstrates that subjects do use the increased preparatory interval to reconfigure the saccadic task-set in advance. However, the critical finding was that the latency switch cost at short cue- target intervals could not be reduced in a similar fashion by foreknowledge (i.e. using a predictable sequence), regardless of whether a prosaccade or antisaccade was required. In fact, the main effect of the predictable sequence was to incur a slight but significant increase in the latency switch costs at both long and short cue-target intervals, compared with the random sequences.

While the latency effects of set-foreknowledge were small, the effects on accuracy were greater. Overall error rate fell significantly from $10 \%$ to $7 \%$ and the accuracy improvement was more pronounced for the more difficult trials, namely those with short cue-target preparatory intervals or antisaccades. However, there was no significant impact on the accuracy switch cost (the difference between the error rates of switched versus repeated saccades).

These results clearly demonstrate that set-foreknowledge does not permit set-reconfiguration in a prosaccade/ antisaccade paradigm. One might argue that the simplest explanation is that the subjects did not avail themselves of foreknowledge, since all trials contained explicit cues and sequence knowledge was not necessary to perform each task. However, the results did show that subjects were using set-foreknowledge to improve their readiness for the upcoming task, since predictability improved accuracy overall, and in particular for the more difficult trials. Thus it seems likely that subjects used working memory to exploit benefits of set-foreknowledge on accuracy, but, surprisingly, could not use set-foreknowledge to reconfigure the task set in advance of the target appearance and thereby reduce latency switch costs.

The prosaccade/antisaccade paradigm is an example of switching between stimulus-response mappings. That is, one task-set demands that response $X$ occur to stimulus $A$ and response $Y$ to stimulus $B$, and the competing taskset reverses the rules, so that response $Y$ occurs to stimulus $A$ and response $X$ to stimulus $B$. Whether these findings of improved accuracy but no benefits to advance reconfiguration generalize to other types of task-switching is unknown. Many other aspects of a trial could be the focus of a switch process (Monsell et al., 2000). In the Stroop test, for example, subjects switch between attending and responding to different dimensions of the stimulus. The stimuli consist of a string of names of colors printed in different colored inks that do not correspond to the color name. In one set of responses the subject names the color of the ink; in the other they read the color name: thus the switch is between whether they attend to the ink color or the printed word. The Stroop test has been an important paradigm for the study of dominance effects in task-switching, where one task is more difficult to perform than the other (Allport et al., 1994; Monsell et al., 2001; Ward et al., 2001; Wylie and Allport, 2000).

We performed a second experiment to see if a similar failure to reduce latency switch costs with task-set foreknowledge was also present in the Stroop paradigm. We had 21 young subjects perform a task-switch study using Stroop stimuli, recording the latency of their verbal responses. We followed a previous study's design in dividing the screen into four quadrants, with one type of response 
A.
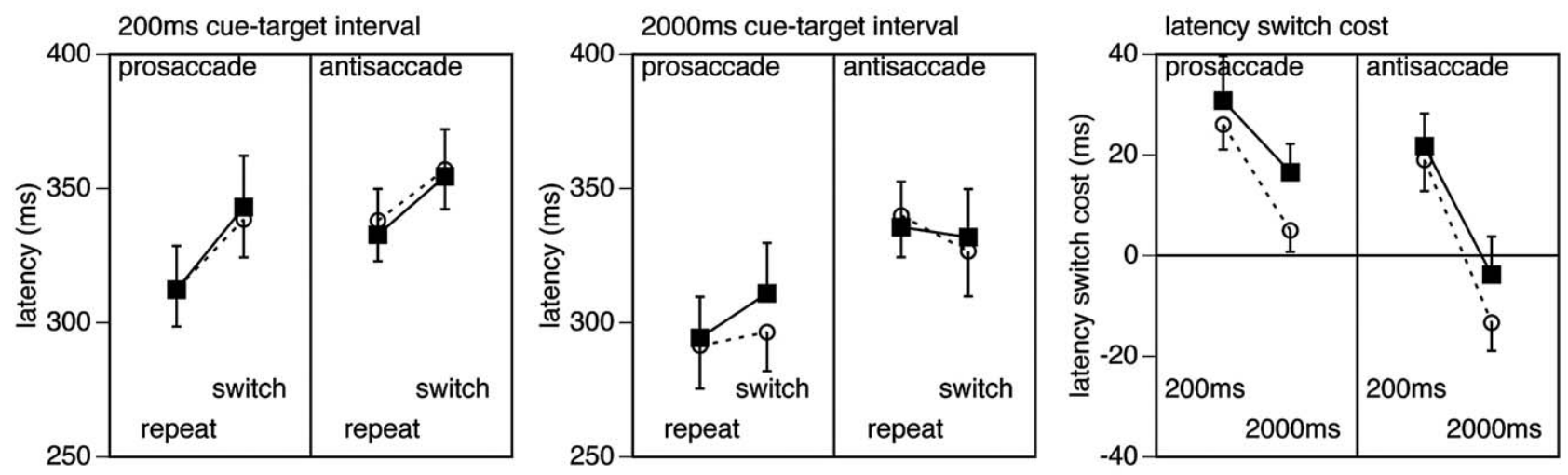

B.
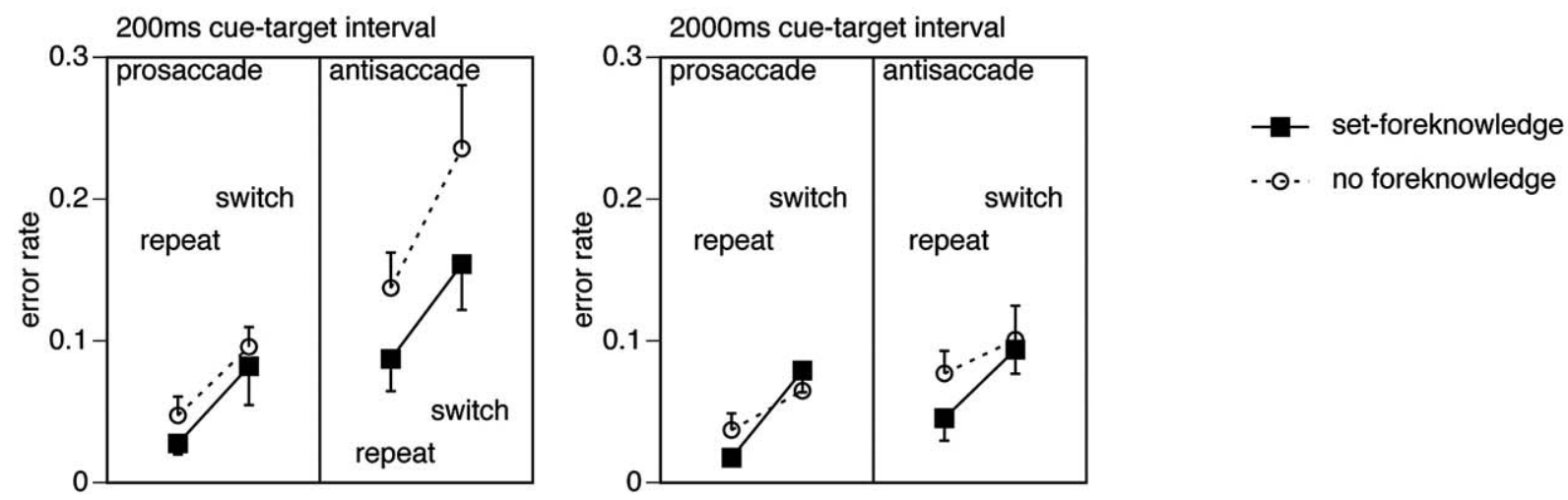

Fig. 1. Set-foreknowledge in a task switching study of prosaccades and antisaccades. Normal subjects performed mixtures of antisaccades and prosaccades, with an instructional cue preceding the target by either $200 \mathrm{~ms}$ or $2000 \mathrm{~ms}$. One set of blocks had a random trial order, the other had a repeating sequence of two prosaccades followed by two antisaccades (with target directions still randomized). (A) Latency effects. If setforeknowledge can trigger advance reconfiguration of a switched trial as effectively as an explicit cue with a long (2000 ms) cue-target interval, then having foreknowledge at the short $(200 \mathrm{~ms})$ cue-target interval should change the results to resemble the no-foreknowledge condition with a long cue-target interval. However the left graph shows that foreknowledge has no effect on the latencies of switched trials with short cue-target intervals. The right graph displays the switch costs (switched minus repeated latencies). A long cue target interval of $2000 \mathrm{~ms}$ significantly reduces switch costs but having set-foreknowledge at the $200 \mathrm{~ms}$ cue-target interval does not. In fact, at both cue-target intervals the switch costs of both types of saccades are slightly but consistently elevated when foreknowledge is available. (B) Accuracy effects. While there is no significant effect of foreknowledge on switching costs, there is reduced error rate for antisaccades and saccades with short cue-target intervals. Error bars show 1 standard error in all graphs.

being required if the stimulus appeared in a top quadrant and the other if it appeared in a bottom quadrant (Wylie and Allport, 2000). In one type of block, the stimuli moved from one quadrant to its neighbor in a predictable clockwise fashion, with a switch every two trials. Because, stimulus location served as the instructional cue, the cuetarget interval was zero. In the other type of block, the stimulus appeared in a quarter chosen at random, but the outline of the quadrant flashed 2000 ms before the stimulus appeared, providing an explicit instruction with a long cue-target interval. If set-foreknowledge served as an effective means for allowing set-reconfiguration in advance of the stimulus, then the latency switch costs should be identical in the two types of blocks. If not, then the latency switch costs should be greater in the predictable series with no cue-target interval. As with the prosaccade/antisaccade paradigm, the Stroop experiment showed that latency switch costs were significantly greater with the short cue-target interval, despite the presence of a predictable sequence (Fig. 2).
The results of these two experiments are contrary to assumptions made in many task-switching studies (Nieuwenhuis and Monsell, 2002) that set-foreknowledge can remove the costs of advance reconfiguration from the residual switch differences being studied. However, they are consistent with a number of other investigations that have directly compared random and predictable sequences for set-switching between attending to different dimensions of a visual stimulus (Dreher et al., 2002; Koch, 2005; Lien et al., 2003; Sohn and Anderson, 2001; Sohn and Carlson, 2000; Tornay and Milan, 2001). Like us, these investigators have shown either no change or even slightly increased latency switch costs with set-foreknowledge, despite similar improvements in general accuracy (Tornay and Milan, 2001), general reductions in response latency (Dreher et al., 2002; Sohn and Carlson, 2000), or reduced latencies in other task effects (Lien et al., 2003). Taking a slightly different approach, one study compared predictable sequences with and without explicit cues (Koch, 2003), rather than the strategy followed by the other stud- 

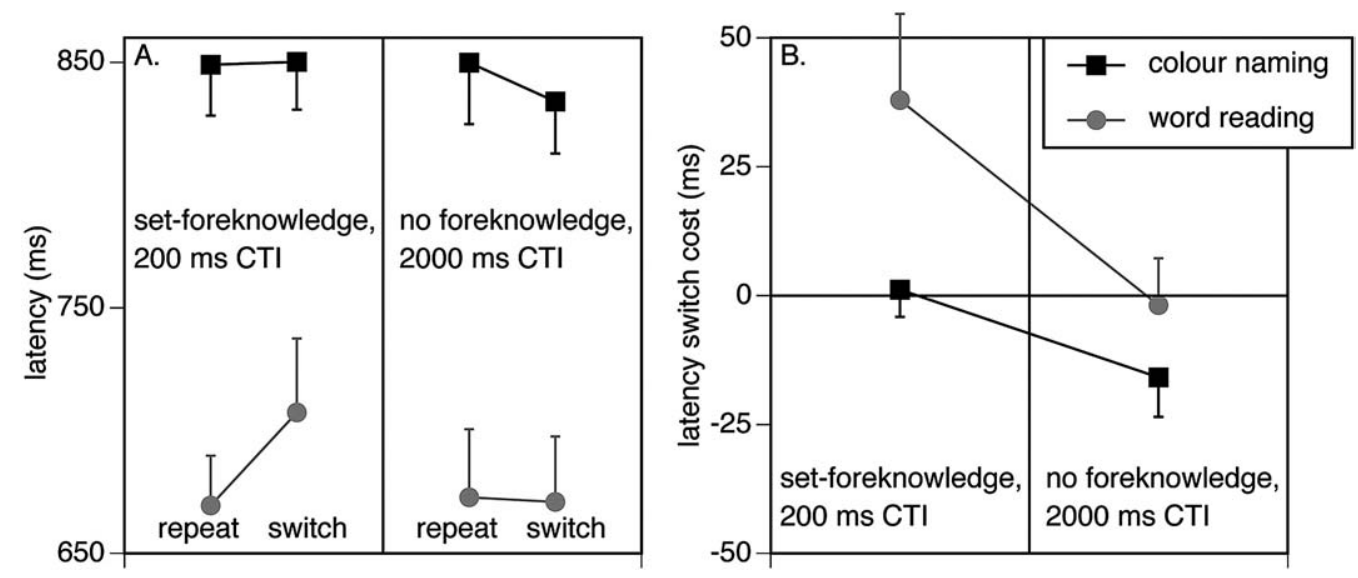

Fig. 2. Set-foreknowledge in a task switching study with Stroop stimuli. (A) Group means of the median latencies of 21 normal subjects. (B) Latency switch costs (switched minus repeated latencies). If set-foreknowledge was as effective as a cue with a long cue-target interval (CTI) in reconfiguring the Stroop task-set, then the switch costs with set-foreknowledge at $200 \mathrm{~ms}$ CTI should be equivalent to the switch costs with no foreknowledge but a long 2000 ms CTI. The results show that, as with the saccade study, switch costs at short CTI are elevated compared with costs at long CTI, despite the availability of foreknowledge. Error bars show 1 standard error.

ies of comparing explicitly cued trials with and without predictable sequences. This found that advance reconfiguration between the dimensions of the stimulus attended was far more efficient when the predictable sequence was accompanied by an explicit cue. Likewise, others have shown that the learning of task-set sequences reduces only general reaction times and not task switch costs (Heuer et al., 2001; Koch, 2001).

The conclusion from all of these studies is that setforeknowledge has specific benefits and specific inefficacies. It allows one to improve accuracy and sometimes latency in a general manner, indicating that the subject can use set-foreknowledge to improve their 'readiness' for accurately executing responses. However, set-foreknowledge does not allow a subject to reconfigure task-sets during a set-switch, in advance of the imperative stimulus, in the same manner that a long preparatory period after an explicit cue does. Hence set-reconfiguration appears to be a relatively impenetrable cognitive operation to the effects of set-foreknowledge in working memory. Koch (2003) views this as a failure of internal cues to trigger the retrieval of task-specific stimulus-response rules from long-term memory, which others propose to be the chief source of intentional switch-costs (Mayr and Kliegl, 2000). This failure to decrease latency stands in sharp distinction to the reductions in latency possible with task-foreknowledge (Kingstone and Klein, 1993; Moschner and Zangemeister, 1993; Schiller et al., 2004), which indicate that significant aspects of response (rather than set) preparation are modifiable by foreknowledge. Interestingly, while pre-cueing but not set-foreknowledge is effective at preparing set reconfiguration, response-foreknowledge may be more effective than certain forms of pre-cueing for response preparation (Schiller et al., 2004).

\section{Foreknowledge in other fields}

The findings reviewed above illustrate important behavioral differences between the effects of different types of foreknowledge. The value of a distinct taxonomy of foreknowledge is also being reflected in the fields of neurophysiology and functional neuroimaging. In terms of timing, neural recordings show that anticipatory activity in the lateral intraparietal area correlates with the probability of a 'go' signal that triggers saccades (Janssen and Shadlen, 2005). More specific studies of timing-foreknowledge report a progressive activation of neurons in the pre-supplementary motor area while awaiting the predictable onset of a target, which the authors hypothesize may reflect 'processing of visual instructions in working memory or . . the preparation of the subsequent movement' (Akkal et al., 2004). Of note, this cortical region has been shown to activate in other time estimation tasks during positron emission tomographic studies of human subjects (Macar et al., 2002). In contrast, an event-related functional magnetic resonance imaging (fMRI) study of task-switching reported that contrasts between set-foreknowledge and no foreknowledge revealed activations in lateral prefrontal, temporal and posterior parietal cortex, rather than the presupplementary motor area (Sohn et al., 2000). The activity in lateral prefrontal cortex is consistent with the engagement of working memory by foreknowledge. One fMRI study has assessed both timing-foreknowledge and setforeknowledge independently (Dreher et al., 2002). Timing-foreknowledge was associated with activation in the middle and superior frontal gyri while set-foreknowledge was associated with activation in the anterior medial prefrontal and posterior cingulate cortex. Although preliminary, these findings imply that different neural networks mediate the effects of timing- and set-foreknowledge, stressing the importance of such behavioral distinctions.

What about task-foreknowledge? Related studies on the prior probabilities of target (and therefore saccadic) location have shown that the pre-target activity of neurons in the superior colliculus varies in relation to the probability of a target, and that this activity correlates with saccadic latency and can even predict the occurrence of anticipatory 
saccades (Basso and Wurtz, 1998; Dorris and Munoz, 1998). Thus advance knowledge can prepare a motor response program at this brainstem level. At the cortical level, fMRI work showing preparatory activity related to a saccadic cue in the frontal but not the parietal eye field (Connolly et al., 2002, 2005), would imply that task-foreknowledge effects on saccades may be found in the frontal eye field. Indeed an fMRI study of prosaccades contrasting predictable timing and predictable target direction showed increased activity in the frontal eye fields with both taskand timing-foreknowledge (Gagnon et al., 2002). Of note, this study found that the difference between these two types of foreknowledge lay not in cortex but in the basal ganglia, with more activity in the putamen and globus pallidus for timing-foreknowledge, and more activity in the caudate for task-(direction)-foreknowledge.

Foreknowledge effects are also beginning to be investigated in neuropsychology, but almost exclusively with paradigms that provide complete timing- and task-foreknowledge. Patients with Huntington's disease fail to generate anticipatory saccades under these completely predictable circumstances (Lasker and Zee, 1997; Tian et al., 1991). In contrast, on a nearly identical paradigm, subjects with schizophrenia actually made saccades with shorter latencies and smaller amplitudes than controls (McDowell et al., 1996), implying an abnormal use of foreknowledge, rather than an inability to exploit it. A similar finding was reported by another study, where with task-foreknowledge schizophrenic subjects made more anticipatory saccades with shorter latencies than control subjects, and some of these parameters correlated with scores on the Wisconsin Card Sort test and the positive and negative symptoms scale (Karoumi et al., 1998). However, these abnormalities were not replicated in a third study with a very similar paradigm (Krebs et al., 2001). An increased saccadic hypometria under these predictable conditions has been interpreted as defective internal ocular motor programming in schizophrenia (Krebs et al., 2001).

Predictive saccades have been studied in Alzheimer's disease, but the specific effects due to foreknowledge are not clear (Abel et al., 2002). Alcohol does not appear to impair the use of task-foreknowledge in predictive saccades (Wegner and Fahle, 1999). Frontal lobe lesions reduce the frequency of anticipatory saccades in the same predictive paradigm (Rivaud et al., 1994), while lesions of the posterior internal capsule, affecting projections from the lateral intraparietal area to the superior colliculus, reduce the amplitude of reflexive but not predictive saccades. These results have been interpreted as evidence that task-foreknowledge in the saccadic system is mediated by circuitry involving frontal cortex, basal ganglia and brainstem. What about stimulus-foreknowledge? The study of attentional allocation to words mentioned above also tested schizophrenic patients. It found that these subjects did not show the modulation of stimulus processing by prior probabilities that was displayed by healthy controls (Myles-Worsley et al., 1991).

These studies suggest the potential value of investigations of foreknowledge effects in dissociating systems in- volved in adaptive behavior and specifying the systems affected in neuropathological states. Clearly, as the studies on set foreknowledge illustrate, the effects of foreknowledge cannot be predicted or assumed in advance, and more work is required to delineate the specific effects of different types of foreknowledge. Further work on partially predictive paradigms will help elucidate which other cognitive operations in the generation of responses to stimuli can potentially benefit from the deployment of foreknowledge in working memory, the neural basis of these operations, and their status in the brain-injured patient.

\section{REFERENCES}

Abel L, Unverzagt F, Yee R (2002) Effects of stimulus predictability and interstimulus gap on saccades in Alzheimer's disease. Dement Geriatr Cogn Disord 13(4):235-243.

Akkal D, Escola L, Bioulac B, Burbaud P (2004) Time predictability modulates pre-supplementary motor area neuronal activity. Neuroreport 15:1283-1286.

Allport A, Styles E, Hsieh S (1994) Shifting intentional set: exploring the dynamic control of tasks. In: Attention and PerformanceV (Umiltà C, Moscovitch M, eds), pp 421-452. Hillsdale NJ: Erlbaum.

Baddeley A (1992) Working memory. Science 255:556-559.

Barton J, Greenzang C, Hefter R, Edelman J, Manoach D (2005) Switching, plasticity, and prediction in a saccadic task-switch paradigm. Exp Brain Res 2005;epub:1-12.

Basso M, Wurtz R (1998) Modulation of neuronal activity in superior colliculus by changes in target probability. J Neurosci 18(8):75197534.

Carpenter R, Williams M (1995) Neural computation of log likelihood in control of saccadic eye movements. Nature 377:59-62.

Connolly J, Goodale M, Goltz H, Munoz D (2005) fMRI activation in the human frontal eye field is correlated with saccadic reaction time. J Neurophysiol 94:605-611.

Connolly J, Goodale M, Menon R, Munoz D (2002) Human fMRI evidence for the neural correlates of preparatory set. Nat Neurosci 5:1345-1352.

Dorris M, Munoz D (1998) Saccadic probability influences motor preparation signals and time to saccadic inhibition. J Neurosci 18(17): 7015-7026.

Dreher JC, Koechlin E, Ali SO, Grafman J (2002) The roles of timing and task order during task switching. Neuroimage 17(1):95-109.

Dreisbach G, Haider H, Kluwe RH (2002) Preparatory processes in the task-switching paradigm: evidence from the use of probability cues. J Exp Psychol Learn Mem Cogn 28(3):468-483.

Gagnon D, O'Driscoll G, Petrides M, Pike G (2002) The effect of spatial and temporal information on saccades and neural activity in oculomotor structures. Brain 125:123-139.

Heuer H, Schmitdke V, Kleinsorge T (2001) Implicit learning of sequences of tasks. J Exp Psychol Learning Mem Cogn 27:967-983.

Janssen P, Shadlen M (2005) A representation of the hazard rate of elapsed time in macaque area LIP. Nat Neurosci 8:234-241.

Kalesnykas R, Hallett P (1987) The differentiation of visually guided and anticipatory saccades in gap and overlap paradigms. Exp Brain Res 68:115-121.

Karoumi B, Ventre-Dominey J, Dalery J (1998) Predictive saccade behaviour is enhanced in schizophrenia. Cognition 68:B81-B91.

Kingstone A, Klein R (1993) What are human express saccades? Percept Psychophys 54:260-273.

Koch I (2001) Automatic and intentional activation of task sets. J Exp Psychol Learning Mem Cogn 27:1474-1486.

Koch I (2003) The role of external cues for endogenous advance reconfiguration in task switching. Psychon Bull Rev 10(2):488492.

Koch I (2005) Sequential task predictability in task switching. Psychon Bull Rev 12(1):107-112. 
Krebs M-O, Gut-Fayand A, Amado I, Daban C, Bourdel M-C, Poirier M-F, Berthoz A (2001) Impairment of predictive saccades in schizophrenia. Neuroreport 12:465-469.

Lasker A, Zee D (1997) Ocular motor abnormalities in Huntington's disease. Vision Res 37:3639-3645.

Lien M-C, Schweickert R, Proctor R (2003) Task switching and response correspondence in the psychological refractory period paradigm. J Exp Psychol Hum Percept Perform 29:692-712.

Macar F, Lejeune H, Bonnet M, Ferrar A, Pouthas V, Vidal F, Maquet $P(2002)$ Activation of the supplementary motor area and of attentional networks during temporal processing. Exp Brain Res 2002: 475-485.

Mayr U, Kliegl R (2000) Task-set switching and long-term memory retrieval. J Exp Psychol Learning Mem Cogn 26:1124-1140.

McDowell J, Clementz B, Wixted J (1996) Timing and amplitude of saccades during predictive tracking in schizophrenia. Psychophysiology 33:93-101.

Megaw E, Armstrong W (1973) Individual and simultaneous tracking of a step input by the horizontal saccadic eye movement and manual control systems. J Exp Psychol 100:18-28.

Meiran N (1996) Reconfiguration of processing mode prior to task performance. J Exp Psychol Learn Mem Cogn 22:1423-1442.

Meiran N (2000) Modeling cognitive control in task switching. Psychol Res 63:234-249.

Meiran N, Chorev Z, Sapir A (2000) Component processes in task switching. Cogn Psychol 41(3):211-253.

Michard A, Tetard C, Levy-Schoen A (1974) Attent au signal et temps de reáction oculomoteur. Année Psychol 74:378-402.

Monsell S, Taylor T, Murphy K (2001) Naming the color of a word: is it responses or task sets that compete? Memory Cogn 29(1): 137-151.

Monsell S, Yeung N, Azuma R (2000) Reconfiguration of task-set: is it easier to switch to the weaker task? Psychol Res 63:250-264.

Moschner C, Zangemeister W (1993) Preview control of gaze saccades: efficacy of prediction modulates eye-head interaction during human saccades. Neurol Res 15:417-432.

Myles-Worsley M, Johnston W, Wender P (1991) Spontaneous selective attention in schizophrenia. Psychiatry Res 39:167-179.

Nieuwenhuis S, Monsell S (2002) Residual costs in task switching: testing the failure-to-engage hypothesis. Psychon Bull Rev 9(1): $86-92$.
Paré M, Munoz D (1996) Saccadic reaction times in the monkey: advance preparation of oculomotor programs is primarily responsible for express saccade occurrence. J Neurophysiol 76(6):36663681.

Rivaud S, Müri R, Gaymard B, Vermesch A, Pierrot-Deseilligny C (1994) Eye movement disorders after frontal eye field lesions in humans. Exp Brain Res 102:110-120.

Rogers RD, Monsell S (1995) Costs of a predictable switch between simple cognitive tasks. J Exp Psychol Gen 124:207-231.

Saslow M (1967) Latency for saccadic eye movement. J Opt Soc Am 57:1030-1033.

Schiller P, Haushofer J, Kendall G (2004) How do target predictability and precueing affect the production of express saccades in monkeys? Eur J Neurosci 19:1963-1968.

Sohn M-H, Anderson J (2001) Task preparation and task repetition: two-component model of task-switching. J Exp Psychol Gen 130: 764-778.

Sohn M-H, Carlson R (2000) Effects of repetition and foreknowledge in task-set reconfiguration. J Exp Psychol Learn Mem Cogn 26: 1445-1460.

Sohn M-H, Ursu S, Anderson J, Stenger V, Carter C (2000) The role of prefrontal cortex and posterior parietal cortex in task switching. Proc Natl Acad Sci U S A 97:13448-13453.

Tian J, Zee D, Lasker A, Folstein S (1991) Saccades in Huntington's disease: predictive tracking and interaction between release of fixation and initiation of saccades. Neurology 41:875-881.

Tornay FJ, Milan EG (2001) A more complete task-set reconfiguration in random than in predictable task switch. $Q \mathrm{~J}$ Exp Psychol $A$ 54(3):785-803.

Viviani P, Swensson R (1982) Saccadic eye movements to peripherally discriminated targets. J Exp Psychol Hum Percept Perform 8:113-126.

Ward G, Roberts MJ, Phillips LH (2001) Task-switching costs, Stroopcosts, and executive control: a correlational study. Q J Exp Psychol A54(2):491-511.

Wegner A-J, Fahle M (1999) Alcohol and visually guided saccades: gap effect and predictability of target location. Psychopharmacology 146:24-32.

Wylie G, Allport A (2000) Task switching and the measurement of 'switch costs.' Psychol Res 63:212-233. 